\title{
A New Approach of Geodesic Reconstruction for Drusen Segmentation in Eye Fundus Images
}

\author{
Zakaria Ben Sbeh, Laurent D. Cohen*, Senior Member, IEEE, Gérard Mimoun, and Gabriel Coscas
}

\begin{abstract}
Segmentation of bright blobs in an image is an important problem in computer vision and particularly in biomedical imaging. In retinal angiography, segmentation of drusen, a yellowish deposit located on the retina, is a serious challenge in proper diagnosis and prevention of further complications.

Drusen extraction using classic segmentation methods does not lead to good results. We present a new segmentation method based on new transformations we introduced in mathematical morphology. It is based on the search for a new class of regional maxima components of the image. These maxima correspond to the regions inside the drusen.

We present experimental results for drusen extraction using images containing examples having different types and shapes of drusen. We also apply our segmentation technique to two important cases of dynamic sequences of drusen images. The first case is for tracking the average gray level of a particular drusen in a sequence of angiographic images during a fluorescein exam. The second case is for registration and matching of two angiographic images from widely spaced exams in order to characterize the evolution of drusen.
\end{abstract}

Index Terms-Drusen, edge detection, eye fundus angiography, geodesic reconstruction, image segmentation, mathematical morphology, registration.

\section{INTRODUCTION}

W ITH aging, the eye experiences a number of pathologies, one of which is the appearance of macular drusen.

Drusen are yellowish deposits located at the level of the retinal pigmentary epithelium. They first appear around age 50 and with time, may change in number and size. Drusen are considered the initial stage of age-related macular degeneration (ARMD), the major cause of legal blindness in developed countries for people age 50 and above.

Significant complications that involve loss of vision, such as hemorrhages, originate with the appearance of drusen.

The exact mechanism for the appearance of drusen is not known. Nevertheless, the modification of size, confluence and coloring of the drusen seems to be an essential risk factor in developing complications. This paper falls within the application of image processing to digitized retinal angiography with a particular emphasis on automatic segmentation of drusen.

Manuscript received March 7, 2000; revised October 1, 2001. The Associate Editor responsible for coordinating the review of this paper and recommending its publication was W. Niessen. Asterisk indicates corresponding author.

Z. Ben Sbeh is with the Ariane group IT Consulting Company, Paris, France.

*L. D. Cohen is with CEREMADE, Université Paris 9-Dauphine Place du Maréchal de Lattre de Tassigny 75775 Paris cedex 16, France (e-mail: COHEN@Ceremade.dauphine.fr).

G. Mimoun and G. Coscas are with the Eye University of Creteil, 94010 Creteil, France.

Publisher Item Identifier S 0278-0062(01)11141-9.

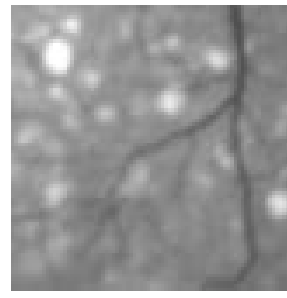

(a)

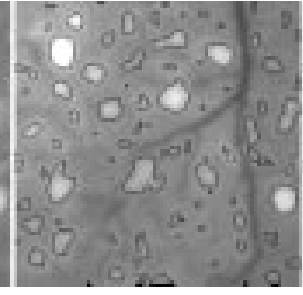

(b)

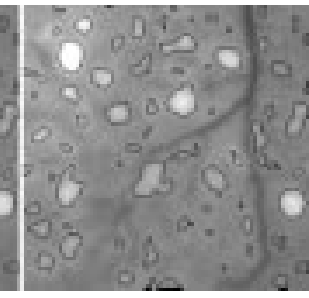

(c)
Fig. 1. Example of drusen segmentation using the $h(x)$-maxima of order $\beta$. (a) Original image, (b) segmentation, and (c) using geometric constraints. Notice in (b) some small regions which are removed in (c).

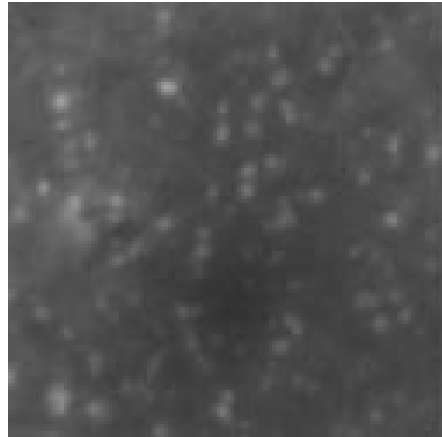

(a)

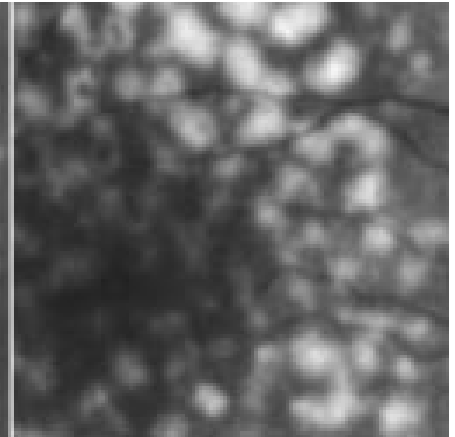

(b)
Fig. 2. (a) Hard drusen. (b) Soft drusen.

Little research has been undertaken in the field of the segmentation and quantization of pathology in retinal ophthalmology. Among these works, one finds the contribution of [27] on the quantification of the gray levels according to the macular types of drusen. The principle used is to digitize an angiography sequence. Image registration is carried out using the initial green stereotype of the vascular tree. Drusen of the sequence are then segmented and tracked manually and then compared using photometry software. The results of this experimental study highlighted the average appearance of the gray levels of the various types of drusen. In spite of the possibility of quantification that it permits, this method suffers from two major handicaps: manual segmentation and manual registration.

The use of traditional segmentation methods generally poses problems due to the nature of images as well as to the aspect of drusen [1]. The single information that we have concerning our problem of drusen detection can be summarized as follows: Drusen are bright spots arising from the background of retinal angiography images. They have nonhomogeneous intensity with a round shape in the best cases (see Figs. 1 and 2). The bright nature of the "spots" enables us to suppose that inside each of these drusen, there is at least a maximum of intensity. 
In this paper, we propose a new segmentation approach based on mathematical morphology. This consists of extracting from the set of traditional regional maxima and minima particular subclasses of connected components (Section III-B). It concerns the maxima and minima of order $\lambda$ (Section III-C). For further study, we showed some mathematical properties of these subclasses in [1].

Also, we introduce a new segmentation tool that we call the $h(x)$-maxima of order $\beta$. It is based on the synthetic construction of an adaptive contrast function $h(x)$ (Section III-F) from maxima and minima of order, respectively, $\lambda, \gamma$. The results of this algorithm are much better than those of the previous approach for $h$-maxima. For that approach it is difficult to get an automatic choice of the $h$ contrast constant and we propose here two ways of defining an automatic value for $h$ (Sections III-E and III-F). The resulting image is like the original image where the background has been removed and it lets appear only those blobs that contain regional maxima.

In Fig. 1, we illustrate an example of drusen segmentation obtained by our segmentation method. In Fig. 1(b), a segmentation using the morphological transformation $f-\delta_{f, \beta}^{\infty}(f(x)-h(x))$ is shown. In Fig. 1(c) The image of the same transformation, under constraints (see Section III-G-3) is shown.

The structure of the paper is as follows: Section II presents the medical background of drusen in angiographic images and underlines the importance of their study. In Section III-A, we present briefly some classic segmentation methods and show that they fail to give satisfying results. After giving some background to Mathematical Morphology in Section III-B, we introduce the notion of Maxima and $h$-maxima of order $\lambda$ in Sections III-C and III-D. We use these notions to find automatically the $h$ contrast parameter in Section III-E to get a first good segmentation of drusen. This method is improved in Section III-F, where we introduce the Adaptive contrast parameter $h(x)$. The method has been shown to be very efficient on various kinds of drusen images. In Section IV, we show how we applied our segmentation tool to track drusen, first during a fluorescein examination and then for the comparison and the mapping of drusen in two examinations taken on two different dates. This is relevant both for the application to quantitative and qualitative study of the evolution of drusen with age. Finally, we give in appendix more background in Mathematical Morphology.

\section{DRUSEN IN ANGIOGRAPHIC IMAGES}

Initiated in the 1970 s by Dallow, digitized retinal angiography is used increasingly in ophthalmology. Its principle consists of replacing traditional photographic images by digitized images. Currently, images of sizes $512 \times 512,768 \times 512$, $1024 \times 1024$ pixels are used with 256 gray levels.

\section{A. Some Pathologies of the Visual System}

Many pathologies of the visual system are due to cellular aging. There are two categories of cellular systems.

- Cellular System With Division: Their speed of renewal is considerable and the cellular system is replaced between one to six days (example: skin, scalp, cornea, etc.).
- Cellular System Without Division: On the contrary, this consists of cells not presenting division after their initial development, such as for example the cells of the neurosensory retina. If the quickly renewed system is surrounded by two systems which do not divide, secondary deteriorations can occur. For example, the presence of waste coming from the system with division and accumulating within the system which does not present division.

A pathology often encountered for older subjects is ARMD. ARMD appears as complications in the macular area. It occurs at age about 50 years and often presents as a deformation and distortion of the images and in particular of the straight lines and a loss of acuity with feeling of hole or scotome in the visual field. Angiographic anomalies include presence of drusen, appearance of subretinal neovessels (SRNV) or choroidal of visible type with sharp edges or occult type with blurred edges, hemorrhages (membrane) or separation of the pigmentary epithelium.

The long-term monitoring of effects such as the diabetic retinopathy or the ARMD, has been recently facilitated by registration and superposition of the images [28]. Our contribution is to go further in the automation of the user's task in the context of drusen segmentation.

\section{B. Drusen}

Drusen constitute the initial signs of the ARMD. They are yellowish of round shape, with hyper-fluorescent spots and are discovered with the examination of the retina in retinal angiography in fluorescence. They correspond to deposits of extra-cellular material located between the basal membrane of the pigmentary epithelium and the collagenous intern layers of Bruch's membrane. Various types of drusen have been described (see Fig. 2):

- Hard Drusen: In optical microscopy, hard drusen appear round, small, of a diameter lower than $50 \mu \mathrm{m}$ with sharp edges [12], [30]. In electronic microscopy, the hard drusen consist of a finely granulous hyaline material [30]. They are interpreted as a metabolic disorder localized in the pigmentary epithelium.

- Soft Drusen: In optical microscopy, they have a structure in the shape of a dome, are larger $(250 \mu \mathrm{m})$ and their contours are blurred [30]. In electronic microscopy, soft drusen consist of a pale amorphous material, comprising membrane remains. Authors of [12], [39] interpret them as a diffuse abnormal operation of the pigmentary epithelium.

- Other Drusen: Mixed drusen: are found between these two extrems. They are round, composed of a combination of membrane remains and residues of hyaline material. They may be derived from hard drusen and transformed into soft drusen [30].

Basal laminar drusen: correspond to a nodular and diffuse thickening of the basal membrane of the pigmentary epithelium. This type of drusen seems to derive from a process different from other drusen [17].

A majority of authors propose epithelial theories: either the cell of the pigmentary epithelium is transformed directly into a 
drusenoidal substance (transformation theory), or the secretion of these cells forms drusen by deposit (deposits theory).

\section{Drusen Evolution}

The evolution of drusen is very variable. They can get joined together, disappear, transform from one type to another or become complicated to neovessels. The prognosis of each type of drusen varies according to the respective risks of neovascular complications. Thus, hard drusen rarely become complicated to neovessels. The soft ones are associated in a way that is statistically significant with subretinal macular neovessels (SRNV) [19]. The pseudoreticular have a bad prognosis. Drusen are strongly related to the early stages of the ARMD, when the visual acuteness is normal before the appearance of complications. Treatment should be considered at this stage.

To prejudge and quantify the natural evolution and the modification of the aspect and number of drusen and the effectiveness of the laser treatment, it is obvious that a quantifiable objective system, ideally automatic, is not only desirable but is impossible to circumvent. The goal of this paper is the development of algorithms for automatic drusen segmentation.

\section{Automatic Segmentation of DRUSEN}

After noting some directions among several that were explored without satisfactory results, we introduce our method based on mathematical morphology.

\section{A. Classic Methods}

The main problems in the classic segmentation methods like thresholding and edge detection are the choice of thresholds, as well as scale factors. Since the images we deal with have a variable contrast and a variable background, even choosing the best threshold, or hysteresis thresholding [4] cannot give good results.

We also tested the watershed method [3], [13], [38] and the main difficulty was in the choice of the minima markers and the oversegmentation of the image [29].

Similarly, drusen may have different sizes in the same image. The authors of [10], [22] use Gaussian Filtering based approaches for blob detection. Notice also that in our case, we are interested only in blobs that are brighter than the background. We did many tests [1] with methods using similar approaches without satisfactory results.

Region Growing and Active Contours: In order to improve edge detection, we also experimented with more complex methods such as region growing and active contours. The region growing methods require germs of initialization, homogeneity criterion, as well as a quality function [15]. We tested this method by taking a certain number of predicates and as starting germs the regional maxima of the image (see Section III-B). The obtained results were not very convincing [1].

The basic idea of Active contours or snakes, consists of placing an initial curve in the neighborhood of the real contour of the object, then deforming it according to a minimization process [16]. This technique has been used extensively and improved during the last few years [9], [20], [26]. Equation (1) represents the energy functional to be minimized in the case of a planar curve $v(s)=(x(s), y(s))$

$$
E(v)=\int_{\Omega}\left[w_{1}\left\|v^{\prime}\right\|^{2}+w_{2}\left\|v^{\prime \prime}\right\|^{2}+P(v(s))\right] d s .
$$

However, deformable contours present some difficulties in the framework of an automatic algorithm, which we summarize as follows. Initialization of the contour must be made near the physical object contour to segment. Energy $E$ admits several local minima, algorithm may converge to a wrong local minimum. This is difficult to define potential function $P$. The method treats only one contour at a time.

Several solutions were proposed to solve these problems. Thus, the balloon model introduced in [8] is less demanding on the initialization, a single point in each drusen would suffice to extract its contour. Geometric implicit active contours [5], [25], [34] make it possible to remove some of the other difficulties. They extend the balloon model and allow not only an automatic management of the changes of topology of contours but also the simultaneous processing of several contours or balloons. However, like the balloon model this model presents also the difficulty of automatic initialization of initial contours. This problem is equivalent to that of the "germs" evoked above in the region growing method. A solution we suggest consists of using regional maxima of the image, noticing the fact that there is at least a regional maxima in each drusen. Once we were on our way to this solution, we found out that the mathematical morphology tools we used to determine the initial regional maxima could be extended to find directly the desired contours. This makes it useless to mix two kinds of methods since we can get the result from the initialization itself. Notice, however, that our results, presented in the next sections could be refined afterwards (if needed) using geodesic active contours [6].

\section{B. Regional Maxima and Minima}

1) Definitions: We give in Appendix some basic definitions of Mathematical morphology. For a complete introduction see [31] and [32]. A function $f$ being given from $\mathbb{R}^{2}$ to $Z$, a chart of $f$ consists of considering its subgraph $G(f)$. This set is similar to a topographic map whose peaks correspond to the bright structures and valleys to the dark structures. The notion of regional maximum and minimum allows the identification of these peaks and valleys.

Definition 1: Given $X \subseteq \mathbb{R}^{2}$, the connected opening [36] at a point $x$ is the set $C_{x}[X]$ defined as the connected component of $X$ containing $x$, if $x \in X$ and $\emptyset$ else.

Definition 2: (Plateau of a function) Let $f$ be a function $f \in \mathcal{F}\left(\mathbb{R}^{2}, Z\right)$, the plateau of $f$ at a point $x$ of $E \subset \mathbb{R}^{2}$ noted $\mathrm{Plt}_{x}(f)$ is the connected component of $E$ containing $x$ and of constant altitude equal to $f(x)$

$$
\operatorname{Plt}_{x}(f)=C_{x}[\{y \in E \mid f(y)=f(x)\}] .
$$

Definition 3: A regional maximum, (respectively, minimum) $M$ of an image $f$ is a plateau, without neighbors of higher (lower) level than $f(M)$. We will note by Max_reg $(f),\left(\min \_r e g(f)\right)$ the set of regional maxima, (minima) of $f$. 
Notice that this definition corresponds to a region of the image and not always a single point. Formally, $M \subseteq \mathbb{R}^{2}$ is a regional maximum (or minimum) of $f$ if and only if there exists $V_{M}$, a neighborhood of $M$ satisfying

$$
\begin{aligned}
\forall_{y} \in V_{M} \backslash\{M\} f(y)<f(M) \\
\quad\left(\text { or } \forall_{y} \in V_{M} \backslash\{M\} f(M)<f(y),\right. \text { respectively). }
\end{aligned}
$$

We note that the concepts of regional maxima and minima are global and nonlocal. One cannot affirm that a connected component is a regional maximum (minimum), by examining only one small arbitrarily selected neighborhood of this component as it is the case for the local maximum concept. This is an advantage since it will not consider points that are seen as local maxima due to a flat neighborhood, but are not truly maxima. Also, only one regional maximum component is detected in a maximum with a flat area instead of many points.

2) Extraction by Markers: A practical way for the extraction of regional maxima and minima consists of using the concept of edges and plateau markers. The advantages of algorithms based on these concepts are their simplicity of implementation and their speed, since they can be written in a parallel way (more details can be found in [1], [2], and [24]) .

Using morphologic dilation, we can define $f_{1}$ and $f_{2}$ the two following functions of $\mathcal{F}\left(\mathbb{R}^{2}, Z\right)$ :

$$
\begin{aligned}
& f_{1}=\min \left(\left[f \oplus g_{H}-f\right], 1\right) \\
& f_{2}=\min \left(\left[\left(f+f_{1}\right) \oplus g_{H}-\left(f+f_{1}\right)\right], 1\right)
\end{aligned}
$$

where $g_{H}$ is a planar structuring element and $f \oplus g_{H}$ is the dilation of $f$ by $g_{H}$ (see Appendix for more details). The binary mask associated with the edges of the plateaus is the set of points such that $f_{1}(x) \neq 0$. Plateau markers correspond to the set of points such that $f_{2}(x) \neq 0$.

Definition 4: A regional maximum is a plateau which has no marker.

3) Extraction by Geodesic Reconstruction: One can also extract the set of regional maxima from an image $f$ by considering the reconstruction from $f$ by geodesic dilation from $(f-1)$ under $f$ (see Appendix, [29] and [37]) and substracting the result from the original image $f$.

Definition 5: The set of regional maxima is defined by

$$
\begin{aligned}
\operatorname{Max\_ reg}(f) & =\left\{x \in \mathbb{R}^{2} /\left(f-\delta_{f}^{+\infty}(f-1)\right)(x)>0\right\} \\
& =\left(f-\delta_{f}^{+\infty}(f-1)\right)_{1}
\end{aligned}
$$

using the notation $F_{h}=\left\{x \in \mathbb{R}^{2} / F(x) \geq h\right\}$ for the $h$ level set of $F$. The equality in (3) is due to the fact that the function has integer values.

Remark 1: The algorithms described previously can be used without any difficulty for the extraction of the regional minima of the image; we just have to replace $f$ by $(-f)$ (or $(255-f)$ to keep the same range of gray levels) and we have $\min \_\operatorname{reg}(f)=$ Max_reg $(-f)$.

The two definitions (markers or geodesic reconstruction) give exactly the same set of points for regional maxima. However, it may be more convenient to use one or the other formula in order

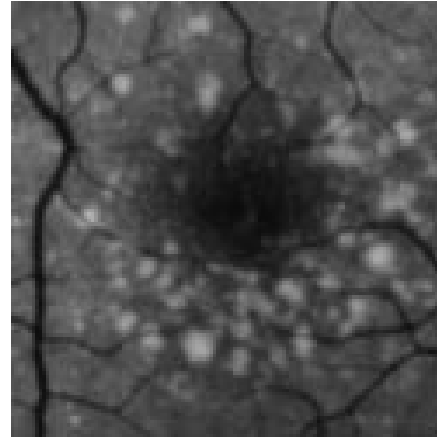

(a)

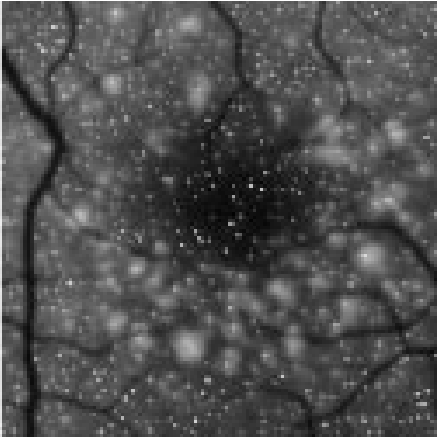

(b)
Fig. 3. (a) the original image. (b) Set of regional maxima.

to show some mathematical properties whether they involve set comparison or distance criteria.

In Fig. 3(b), we show in white the set of regional maxima. We notice that there are far more of them than the number of clear structures present in the original image. We can confirm that each maximum is a peak of intensity in a certain neighborhood which is not necessarily a drusen, or part of drusen. That is why it is natural to find a specific selection criterion of these maxima, in order to keep only the most representative.

\section{Maxima and Minima of Order $\lambda$}

In this section, we define and use a new class of connected components of a given image $f$ depending on a parameter $\lambda>0$ which may be seen as a scale factor.

Definition 6: We call the regional maxima (or maxima) of order $\lambda$ the connected components of the set $\left(f-\delta_{f, \lambda}^{+\infty}(f-1)\right)_{1}$. $\left(\delta_{f, \lambda}\right.$ indicates geodesic dilation under $f$ with respect to a planar structuring element of size $\lambda$, with convention $\delta_{f, 1}(g)=\delta_{f}(g)$ ). We denote by Max_reg ${ }_{\lambda}(f)$ the set of maxima of order $\lambda$.

$$
\text { Max_reg }{ }_{\lambda}(f)=\left\{x \in \mathbb{R}^{2} /\left(f-\delta_{f, \lambda}^{+\infty}(f-1)\right)(x)>0\right\} .
$$

By transposition of the definition of the maxima of order $\lambda$ we can define the set of minima of order $\lambda$ by

$$
\min \_\operatorname{reg}_{\lambda}(f)=\left\{x \in \mathbb{R}^{2} /\left(\varepsilon_{f, \lambda}^{+\infty}(f+1)-f\right)(x)>0\right\}
$$

where $\varepsilon_{f, \lambda}^{+\infty}$ is the geodesic erosion (see Appendix).

We can show that the sets of regional maxima of order $\lambda$, $\left(\operatorname{Max}_{\operatorname{reg}}(f)\right)_{\lambda}$, form a decreasing sequence with regard to inclusion

$$
\begin{aligned}
& \text { Proposition 1: } \forall f \in \mathcal{F}\left(\mathbb{R}^{2}, Z\right) \\
& \quad \text { if } \lambda<\lambda^{\prime} \text {, then Max_reg } \lambda_{\lambda^{\prime}}(f) \subset \operatorname{Max} \_\operatorname{reg}_{\lambda}(f) .
\end{aligned}
$$

As illustrated in Fig. 4, the more the parameter $\lambda$ increases, the less maxima of order $\lambda$ are retained. This is why $\lambda$ can be seen as a parameter for selecting the regional maxima of a given image. The parameter $\lambda$ allows a selection of regional maxima of the image according to their intensity and their interseparation distance (see [1]).

\section{D. h-Maxima}

The previous sections present a way to find starting points for all the bright areas of the image, like drusen. Instead of 


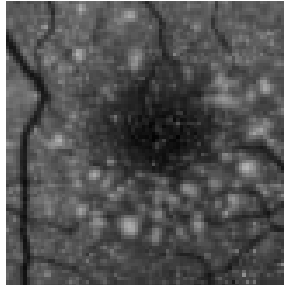

(a)

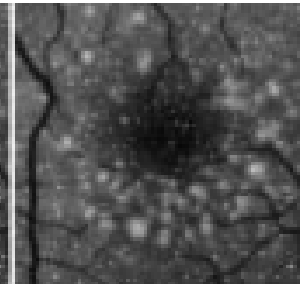

(b)

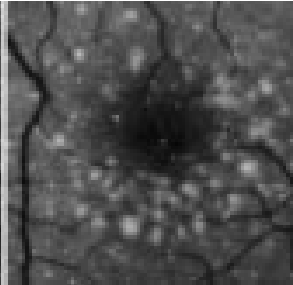

(c)
Fig. 4. In white: Maxima of order. $\lambda$. (a) $\lambda=2$, (b) $\lambda=4$, and (c) $\lambda=7$.

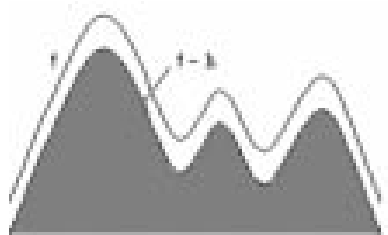

(a)

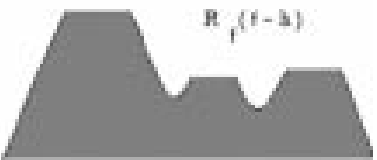

(b)
Fig. 5. (a) Example for a one-dimensional (1-D) profile (a) $f$ and $f-h$. (b) Result of geodesic reconstruction. The three flatten areas show the aspect of the $h$ maxima.

using these points as initialization for a second step, like active contours or balloons [8], we extended the definition of the connected components to get a larger area. Thus, we define a way to find the complete drusen spots with a method that remains based on geodesic reconstruction (dilation or erosion of $f$ ). We will present now the set of $h$-maxima ( $h$-minima can be defined by transposition)

Definition 7: The $h$-maxima of a numerical function $f$ are the connected components of the set $\operatorname{Max}_{h}$

$$
\operatorname{Max}_{h}(f)=\left(f-\delta_{f}^{+\infty}(f-h)\right)_{1} .
$$

The $h$-maxima allow the extraction of the clear structures of the image without any constraint of shape or size. Only the parameter $h$ intervenes. It is related to the height (contrast) of the structures to extract.

Geometrically, the $h$-maxima can be interpreted like the regional maxima of the image $\delta_{f}^{+\infty}(f-h)$ [37] (see Fig. 5, where $R_{f}=\delta_{f}^{+\infty}$ ).

We illustrate in Fig. 6 the principle of our method that has removed the background and kept only the three bright spots.

We note that the $h$-maxima were introduced in order to overcome the problems of noise met with the regional maxima. This concept is more robust and less sensitive to noise. However, the choice of the parameter of contrast $h$ remains to be determined. In order to choose it automatically, we can make some remarks about the total behavior of the algorithm with this parameter. The area of each object extracted depends on the value of parameter $h$. An increase in $h$ is accompanied by a growth of this area. We notice that only the maxima with strong contrast will persist.

By changing the size of the planar structuring elements in the definition of $h$-maxima, we find a new set of connected components which we call $h$-maxima of order $\lambda$.

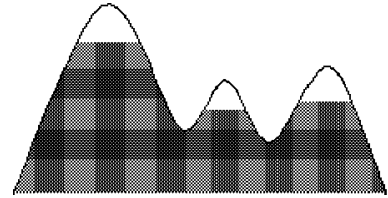

(a)

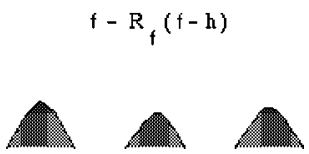

(b)
Fig. 6. Example on a 1-D profile (a) $f$ and $R_{f}(f-h)$ are superimposed. (b) Difference keeps only the bright spots and removes the background.

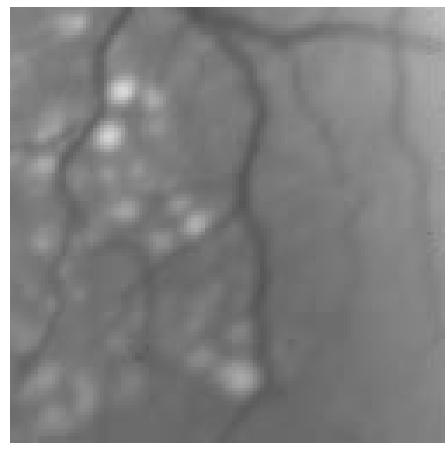

(a)

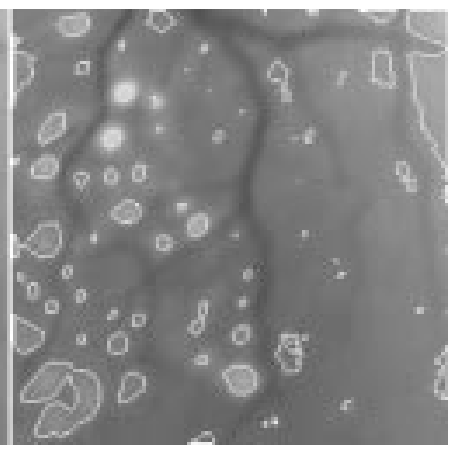

(b)
Fig. 7. (a) Original image. (b) Set $\left(\operatorname{Max}_{h, \lambda}\right)$.

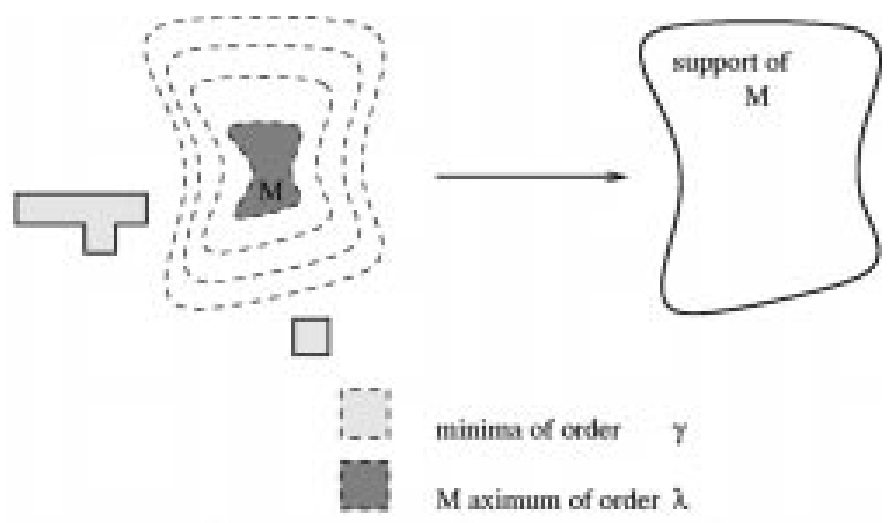

Fig. 8. How to find the nearest minimum of order $\gamma$.

Definition 8: The $h$-maxima of order $\lambda$ of a numerical function $f$ are the connected components of the set $\operatorname{Max}_{h, \lambda}$

$$
\operatorname{Max}_{h, \lambda}(f)=\left(f-\delta_{f, \lambda}^{+\infty}(f-h)\right)_{1} .
$$

In a way similar to maxima of order $\lambda$, the sets of $\left(\operatorname{Max}_{h, \lambda}\right)_{\lambda}$ satisfy the following inclusion:

Proposition 2: $\forall f \in \mathcal{F}\left(\mathbb{R}^{2}, Z\right)$

$$
\text { if } \lambda<\lambda^{\prime} \text {, then } \operatorname{Max}_{h, \lambda^{\prime}}(f) \subset \operatorname{Max}_{h, \lambda}(f) \text {. }
$$

The more the parameter $\lambda$ increases, the more the area of the connected components of $\operatorname{Max}_{h, \lambda}$ decreases. When $\lambda$ grows, some small objects vanish.

We illustrate in Fig. 7 an example of the $\left(\operatorname{Max}_{h, \lambda}\right)$ set, here $\lambda=9$ and $h=30$.

In the first two lines of Fig. 9, we show the evolution of the result with increasing parameter $h$. Here, $h$ appears as a contrast parameter and the result depends much on the choice for this parameter. If $h$ is small, we obtain small regions centered on 


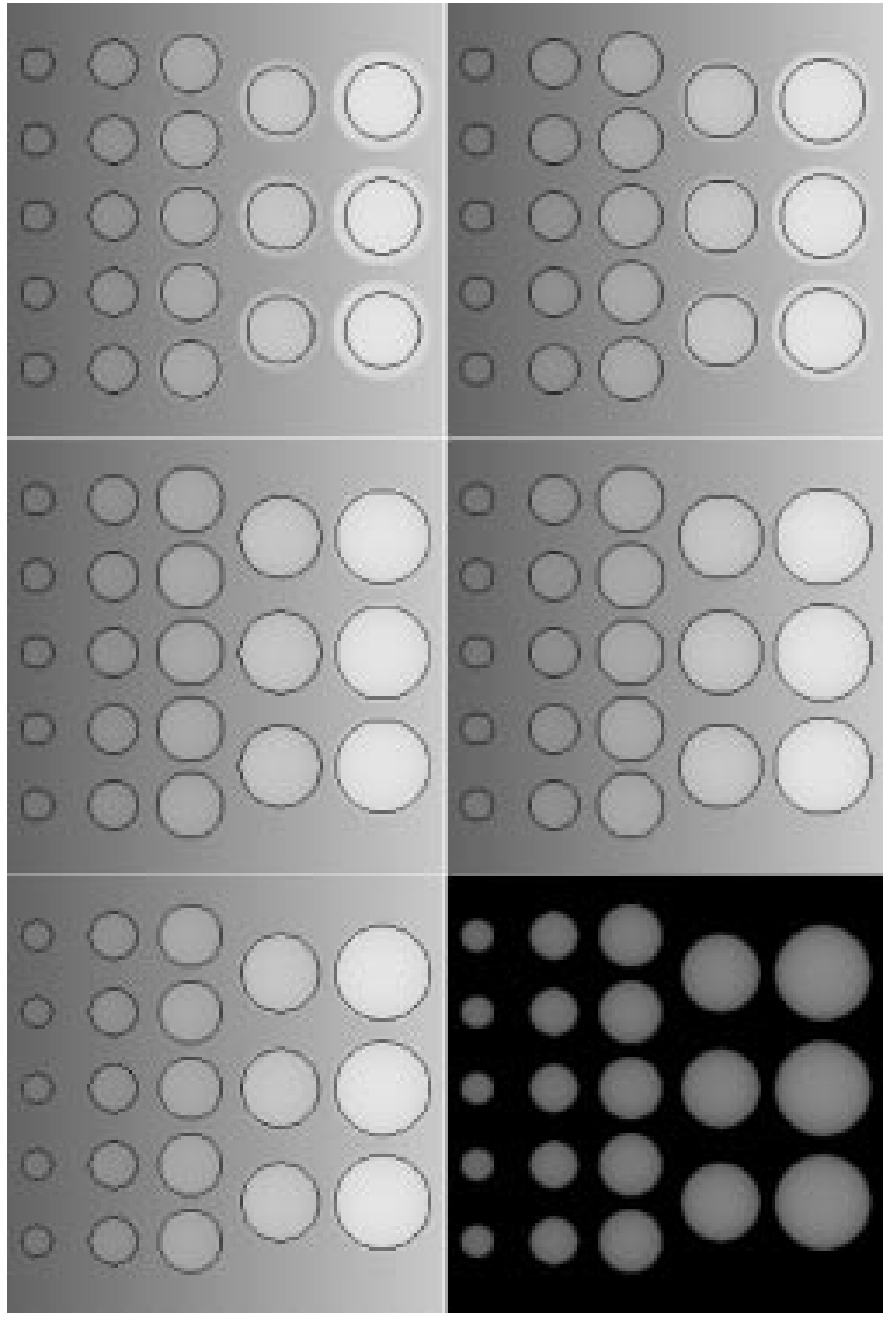

Fig. 9. Test images: blobs are detected in spite of different contrast values. In each blob, the gray level function is defined as ahalf sphere with a maximum in the middle. The background itself has an increasing gray level from left to right. The first two lines show theevolution with increasing constant parameter $h$. On the last line, the result with automatic determination of function $h(x)$ and the transform $f-\delta_{f, \beta}^{+\infty}(f-h)$.

regional maxima of the image. If $h$ increases, the regions grow and can merge together.

\section{E. Automatic Determination of Contrast Parameter $h$}

During the last few years a particular interest was given to the concept of dynamic extremum [11], [21], [22], [35]. In mathematical morphology, we also find this concept in [13], [14], [29], and [33]. In the next section, we define a new concept, which we call "relative dynamics," that we will assign to each one of the maxima of order $\lambda$.

1) Relative Dynamics: The idea of our modeling is based on the fact that one starts from sets of points representative of the clear structures of the image. Then, we try to recover the object marked by a given maximum according to its force of contrast which we measure using its relative dynamics.

The sets which we use are: the maxima of order $\lambda$ and minima of order $\gamma$. We used maxima of order $\lambda$ to mark the centers of drusen and the minima of order $\gamma$ for marking the local background of a drusen near its edges. Thus, according to the distribution of drusen in the image, the doctor will be asked to provide the parameters $\lambda, \gamma$ that will be a kind of characteristic signature of the processed image. This choice would probably be done only once for a set of images that have the same kind of drusen. In practice, we chose parameters $\lambda, \gamma$ verifying: $\lambda \geq \gamma$. We recall that $\lambda, \gamma$ have a significant role in the number of markers of the most representative structures of the image.

Before defining the relative dynamics of a maximum of order $\lambda$, we start by giving a definition of the relative support of a maximum of order $\lambda$. Let $(\lambda, \gamma)$ be two positive numbers of $\mathbb{N}$, $M$ a maximum of order $\lambda$.

Definition 9: The relative support of order $\gamma$ of a maximum $M$, $\operatorname{supp}_{\gamma}(M)$, is the smallest dilated of $M$ with nonempty intersection with $\min \_\mathrm{reg}_{\gamma}^{-}$, where $\min \_\operatorname{reg}-(M)=\left\{p \in \min \_\right.$reg $\left.\gamma(f) / f(p)<f(M)\right\}$.

The following is an algorithm to obtain the relative support

$$
\begin{cases}M_{0} & =M \\ M_{k} & =M_{k-1} \oplus H \\ k_{0} & =\inf \left\{k \in \mathbb{N}, M_{k} \cap \min \_\operatorname{reg}_{\gamma}^{-}(M) \neq \emptyset\right\} \\ \operatorname{supp}_{\gamma}(M) & =M_{k_{0}}\end{cases}
$$

where $H$ is the basic structuring element. This corresponds to a front propagation starting from $M$ and that stops when it meets a minimum. This could be accomplished more efficiently using fast marching simultaneously from all maxima [7].

Definition 10: The relative dynamics of a maximum $M^{\lambda}$ of order $\lambda$ is

$$
\begin{array}{r}
d y n_{\gamma}\left(M^{\lambda}\right)=f\left(M^{\lambda}\right)-\sup \left[f(m) \mid m \in \min \operatorname{reg}_{\gamma}^{-}\left(M^{\lambda}\right)\right. \\
\text { and } \left.m \cap \operatorname{supp}_{\gamma}\left(M^{\lambda}\right) \neq \emptyset\right] .
\end{array}
$$

In some cases, the front of propagation associated with a $M_{\lambda}$ maximum stops after having touched more than one minimum of order $\gamma$, in this case, for the calculation of relative dynamics it is necessary to choose the minimum of order $\gamma$ with maximum intensity.

Remark 2: According to these two definitions we deduce that $\forall M^{\lambda} \in$ Max $r e g_{\lambda}, \forall \gamma \in \mathbb{N}: d y n_{\gamma}\left(M^{\lambda}\right)>0$

2) A First Step Toward Automatic Segmentation of Drusen: One of our first algorithms for the extraction of drusen used the $h$-maximum concept (see Section III-D). However, the results obtained depended too much on the parameter $h$. We, thus, use our concept of $h$-maxima of order $\beta$ (connected components of the set $\left.\left(f-\delta_{f, \beta}^{+\infty}(f-h)\right)_{1}\right)$ and adapt the parameter $h$ to the processed image by giving a value to $\beta$ dependent on the type of drusen used.

A first way to find automatically the value of parameter $h$ is to define it as the average between the maximum and the minimum of relative dynamics of the image:

$$
h=\frac{1}{2}\left(\max _{i}\left[d y n_{\gamma}\left(M_{i}^{\lambda}\right)\right]+\min _{i}\left[d y n_{\gamma}\left(M_{i}^{\lambda}\right)\right]\right)
$$

With this definition of $h$, we obtain rather good results, as shown in the examples of Fig. 12 (middle images). However, there were lacks in the localization and the elimination of the artifacts. This is why we give in the next sections a different way to define a local value of $h$. 


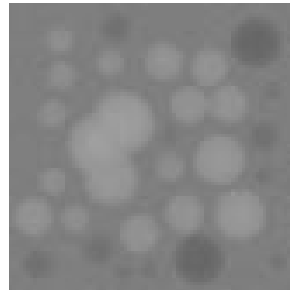

(a)

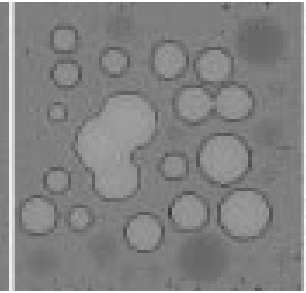

(b)

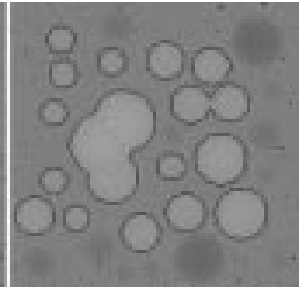

(c)
Fig. 10. Test example. (a) Original image,. (b) Result with automatic determination of constant $h$. (c) Result with automatic determination of function $h(x)$ of (7) with: $\lambda=13, \beta=13$, and $\gamma=21$. Notice for example a difference on the left of the image for the third circle from top which is better localized with $h(x)$ method.

\section{F. Adaptive Contrast Parameter $h(x)$}

In the preceding sections, we saw in various examples that the algorithm of $h$-maxima depends on the value of the parameter $h$ used. We now deal with the following question: in order to get automatic detection of drusen, can we adapt the parameter $h$ to the structures to be extracted?

Our idea consists of adapting locally the parameter $h$ in order to get the bright spots present in the image through the algorithm of $h$-maxima of order $\beta$. Thus, we do not consider $h$ as a constant anymore but as a contrast function $h(x)$ depending on the location on the image.

Definition 11: The $h(x)$-maxima of order $\beta$ of a numerical function $f$ are the connected components of the set

$$
\left(f-\delta_{f, \beta}^{+\infty}(f-h(x))\right)_{1} .
$$

We now give a way to define $h(x)$ in order to detect drusen. If $\left(M_{i}^{\lambda}\right)_{i}$ are the regional maxima of order $\lambda$, let $D_{i}$ represent the intersection between $\operatorname{supp}_{\gamma}\left(M_{i}^{\lambda}\right)$ and the other relative supports of the image $\operatorname{supp}_{\gamma}\left(M_{j}^{\lambda}\right)$. We then define $h(x)$ as follows:

$h(x)= \begin{cases}d y n_{\gamma}\left(M_{i}^{\lambda}\right), & \text { if } x \in \operatorname{supp}_{\gamma}\left(M_{i}^{\lambda}\right) \backslash D_{i} \\ j / x \in \operatorname{supp}_{\gamma}\left(M_{j}^{\lambda}\right)\left[d y n_{\gamma}\left(M_{j}^{\lambda}\right)\right], & \text { if } x \in D_{i} \\ c, & \text { if } \forall i, x \notin \operatorname{supp}_{\gamma}\left(M_{i}^{\lambda}\right) .\end{cases}$

We choose constant $c$ such that $c \leq \min _{i}\left[d y n_{\gamma}\left(M_{i}^{\lambda}\right)\right]$ ). We illustrate first this approach in Fig. 10 with a synthetic example with nonhomogeneous spots on slanted disturbed background. Using $h(x)$ as defined in (7) and defining the constant $c=\min _{i}\left[d y n_{\gamma}\left(M_{i}^{\lambda}\right)\right]$ ), we obtain perfect detection of the clear spots. Notice that, as expected, the dark spots are not extracted. For this simple example traditional methods would be completely inefficient [1].

We show in Fig. 11 the main stages of our approach on a drusen image. In order to simplify calculation of the supports and the relative dynamics, we reduced (just for calculation of $h(x)$ ) each minimum of order $\gamma, m_{\gamma}$ and each maximum of order $\lambda, M_{\lambda}$ to representative points of these connected components (center of gravity). Thus, relative supports are composed of disks.

We see in Fig. 12 illustrations on different kinds of images. The use of a constant $h$ instead of $h(x)$ does not make it possible to emphasize simultaneously all the spots especially for the low values of $h$ (see middle row).

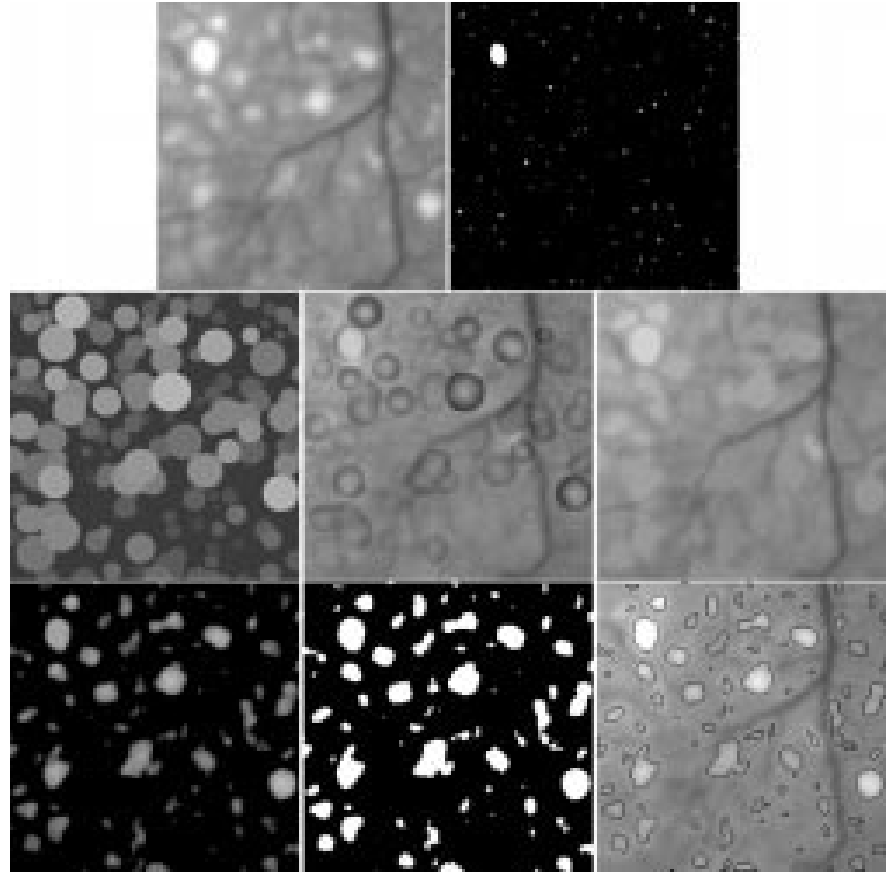

Fig. 11. Main stages of our extraction algorithm: Original image, on the top left; maxima of order $\lambda$ of the original image, on the top right; function $h(x)$ obtained after processing of the maxima and minima of order $\lambda$ of the image, on the left of second row; the image $(f-h(x))$, on the middle of second row; the image $\delta_{f, \beta}^{+\infty}(f-h)$, on the right of second row; the image $f-\delta_{f, \beta}^{+\infty}(f-h)$, on the bottom left; the binarized image of $f-\delta_{f, \beta}^{+\infty}(f-h)$ by thresholding at gray value 1 , on the bottom middle; contours of the previous image superimposed on the original image, on the bottom right.

\section{G. Application to Drusen Segmentation}

In this section, we describe the main stages involved in our algorithm to apply it to drusen segmentation. In order to apply the method introduced in the previous sections, we need a few steps of preprocessing and postprocessing.

1) Preprocessing: Smoothing: Initially, we choose the area of interest of the original image (manual stage carried out by the doctor). We usually focus the search of drusen in the central area of the retina (fovea). Then, in order to reduce the noise, we filter the selected area using a median filter followed by an average filter.

2) Our New Approach for Drusen Segmentation: The next stage consists of building a synthetic image $h(x)$ starting from the set of maxima of order $\lambda$ and minima of order $\gamma$ (generally we take $\gamma \leq \lambda$ ). The choice of the parameter $\lambda$ can be taken as the expected minimal length separating two centers of drusen. This choice is a useful criterion for selecting the regional maxima that can be inside a drusen. Refer to [1] for theoretical results showing that the distance between two regional maxima of order $\lambda$ with different gray levels is always greater than $\lambda$.

On angiography images, drusen correspond to areas with strong contrast. It is the only relevant information we have. This is the place where we use the main algorithm introduced in this paper in Section III-F: the $h(x)$-maxima.

The result obtained at this stage is often accompanied by detection of a certain number of artifacts which will be almost eliminated in the next stage. 

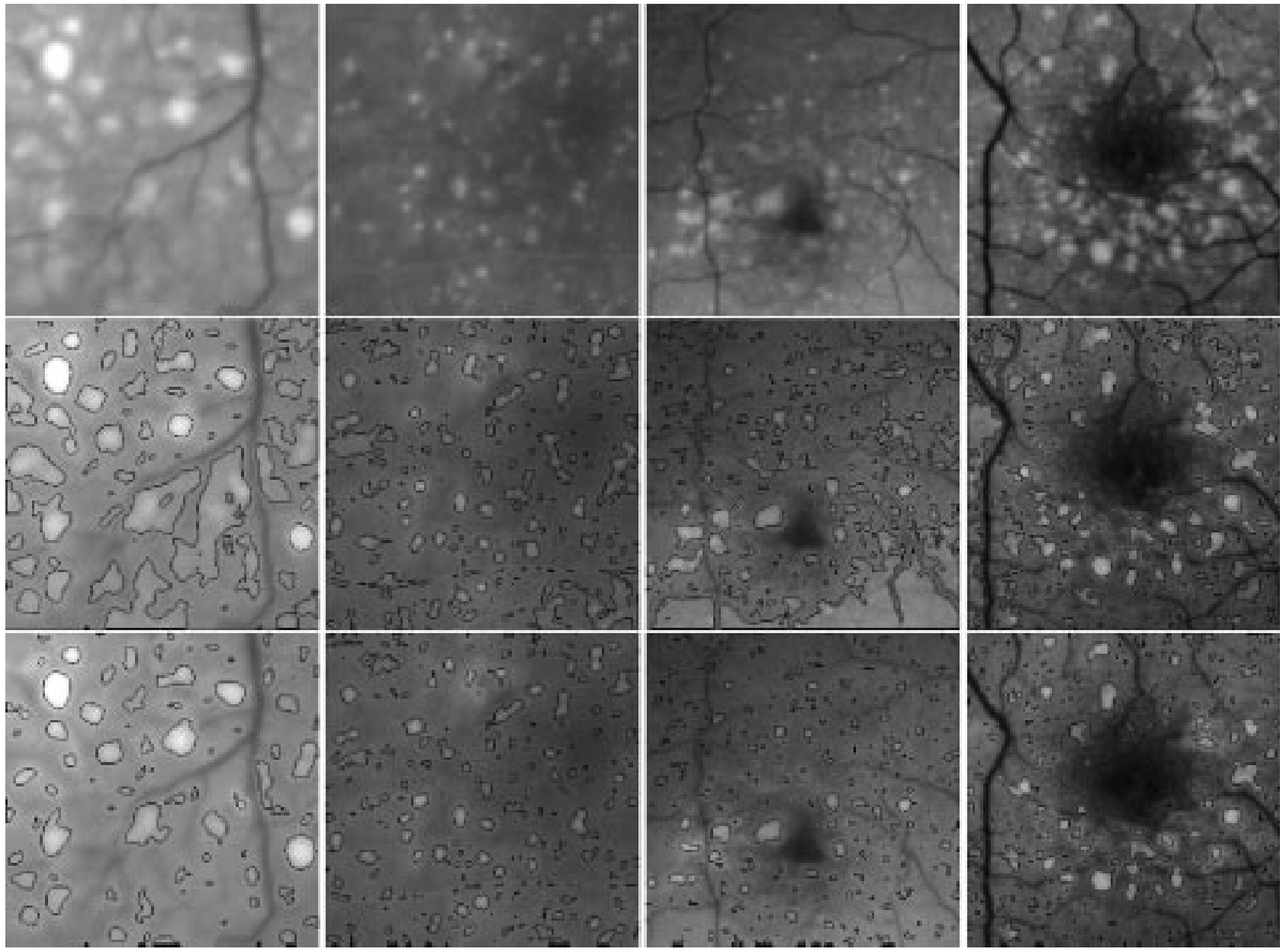

Fig. 12. Different types of real images. (top) Original images. (middle) Our method with constant $h$ from (6). (bottom) Our method with the adaptive function $h(x)$ from (7).

3) Postprocessing: Segmentation Under Constraints: Notice that the results obtained with our method may have to be refined. First there are very small regions (artifact problem) that are detected which we remove using constraints, as described below. Also, in case a very precise contour is needed, active contours may be useful to improve localization of edges (see Section III-A).

In order to keep only the relevant spots and to eliminate a certain number of artifacts, we force constraints on the objects of the final segmentation. These constraints are to be adapted according to the nature of the images processed. For example, in the case of the images obtained with green stereotype, our algorithm will avoid extraction of the blood vessels since they generally do not present a maximum of intensity. On the other hand, in the case of a fluorescein image (see Fig. 14), vessels and drusen have a similar hue. The use of shape and contrast criteria then becomes necessary to differentiate drusen spots from vessels.

- Shape criterion: As a characteristic of the object shape we have chosen the ratio $R=\lambda_{\min } / \lambda_{\max }$ where $\lambda_{\min }, \lambda_{\max }$ are, respectively, the lower and upper eigenvalues of the inertia covariance measure matrix. The ratio $R$ is a measure of the roundness of the object. In the case of drusen, we choose the criteria $s_{1} \leq R$ ( $s_{1}$ is a fixed threshold). This is sufficient to discriminate between drusen and vessels.

- Contrast criterion: In order to overcome the artifact problem in mathematical morphology segmentation, some authors use thresholding and dynamic filtering technique of the $h$-minima ( $h$-maxima) image. As reported by [13], a special care must be done when choosing the $h$ parameter in this case. The author of [13] proposed to choose $h$ close to the minimum dynamics of the structures to be extracted.

Here, we use a similar thresholding approach (contrast criterion) to remove the artifacts of weak contrast. Among the criteria of contrast which one can take into account in this problem of segmentation under constraint, we retain the following criterion over the considered region $R$

$$
C(R)=\left(\max _{R}(g)-\min _{R}(g)\right) .
$$

We used this parameter to eliminate the objects with weak contrast from the result image $g=f-\delta_{f, \beta}^{+\infty}(f-h)$.

- Area criterion: This criterion preserves in the final segmentation image only those objects whose area is higher than a threshold $T$. Thus, small artifacts due to noise will be removed. The threshold has to be chosen rather small since the 


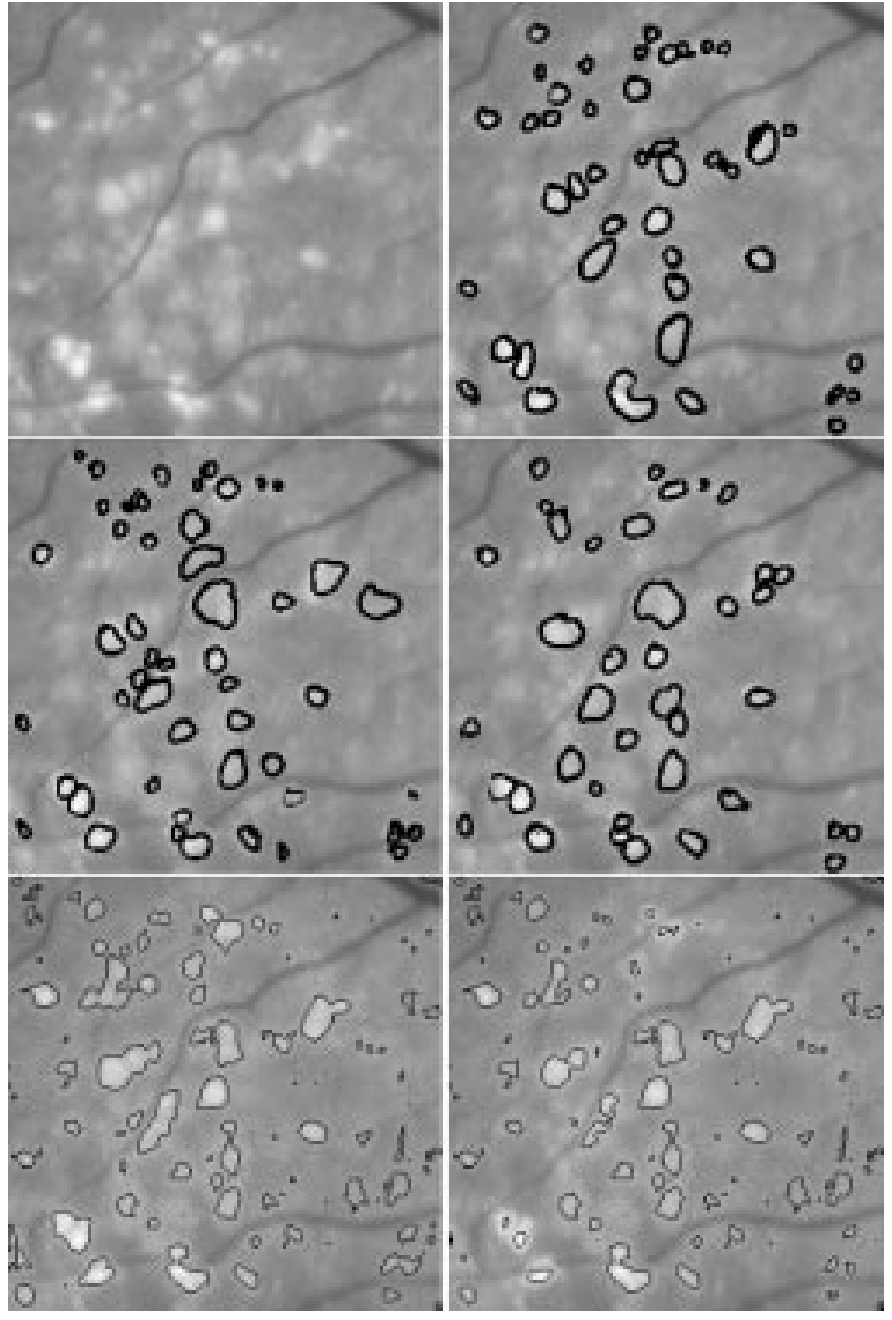

Fig. 13. Manual extraction of drusen illustrates the issue of reproducibility by human drawing versus automatic detection. (top left) Original image (top right and in the second line) Manual drusen segmentation by three different ophthalmologists (last line) Results for automatic drusen segmentation, with constant $h$ (left) and with the adaptive function $h(x)$ (right) with: $\lambda=21$, $\beta=9$, and $\gamma=21$.

only drusen with small surface are round and have high contrast compared to the image background.

4) Results: In Fig. 12, we show results of this method on different kinds of drusen images. This underlines the fact that our approach is efficient for a very large range of images. Results with an adaptive contrast function $h(x)$ are compared with the previous definition of the constant $h$ we used earlier in this paper. We show in the middle row of Fig. 15 examples of segmentations under constraints.

In Fig. 13, we show and compare the hand and automatic segmentation of drusen. The manual segmentation is given by hand drawing by three different ophthalmologists. It can be seen that the differences between these segmentations are often important. This underlines the issue of reproducibility by human drawing and the difficulty of the task at hand. The automatic results are obtained using our method with constant $h$ of (6) and our method with the adaptive function $h(x)$ of (7).

- Computation time: The computation time of our segmentation method depends primarily on the number of maxima of order $\lambda$ which will be processed. In the case of our images, the detection phase of maxima of order $\lambda$, minima of order $\gamma$, construction of the function $h(x)$ takes about $54 \%$ of the total time calculation. The remaining $46 \%$ are to be distributed between the calculation of the function $g=f-\delta_{f, \beta}^{+\infty}(f-h)$ and the postprocessing under constraints of the objects of the image $g$. The constraints stage depends on the number of objects to be processed since for each object, we must test whether or not it satisfies the set of previous criteria. As an indication, on Pentium 133 $\mathrm{MHz}$ (which was used at the time of this work) the complete processing of an image of size $256 \times 256$ containing two objects took about $37 \mathrm{~s}$.

- Objective of drusen segmentation: Once this segmentation has been obtained through the previous steps, there can be various goals of data analysis, like counting drusen and finding their area. This is useful in the context presented in Section IV dealing with drusen tracking and drusen classification.

\section{DRUSEN TRACKING AND ClASSIFICATION}

Tracking drusen during eye examination is very important for ophthalmologists. We propose to describe briefly the stages of the methods which we adopted in order to match drusen in a sequence of retinal angiography. We study two examples of tracking:

- Short term: propagation of fluorescein in a drusen;

- Long term: comparison of the area occupied by drusen at two different examination dates (time between them could be months or years).

In this section, we describe how we can use our segmentation method (of Section III-F) on a sequence of images and how it can contribute to the medical diagnosis of drusen during a fluorescein examination.

\section{A. Registration Problem}

Matching shapes and Image Registration are often used in medical image processing. They consist of superimposing images by the mapping of common characteristic elements. The utility of matching in digital ophthalmology is not to prove. Indeed, it is enough to know that during an examination of retinal angiography, the patient does not cease moving the treated eye, which lets any exploitation of the acquired images almost impossible for sequential analysis. Currently, it is not possible in the medical protocol of the fluorescein examination, nor that with the green of indocyanine to immobilize the eye treated by an anaesthetic product throughout examination, because of danger.

Concerning the problem of image registration in ophthalmology, some progress is reported in [18] who proposed an automatic geometrical method to perform the registration. The author dealt with a pair formed of an image in green light or blue and of an image at the early time of fluorescein injection. The technique starts by detecting the points of bifurcation of the vascular structure of the retinal images to match. These junction points are then matched to find the best affine transform. 


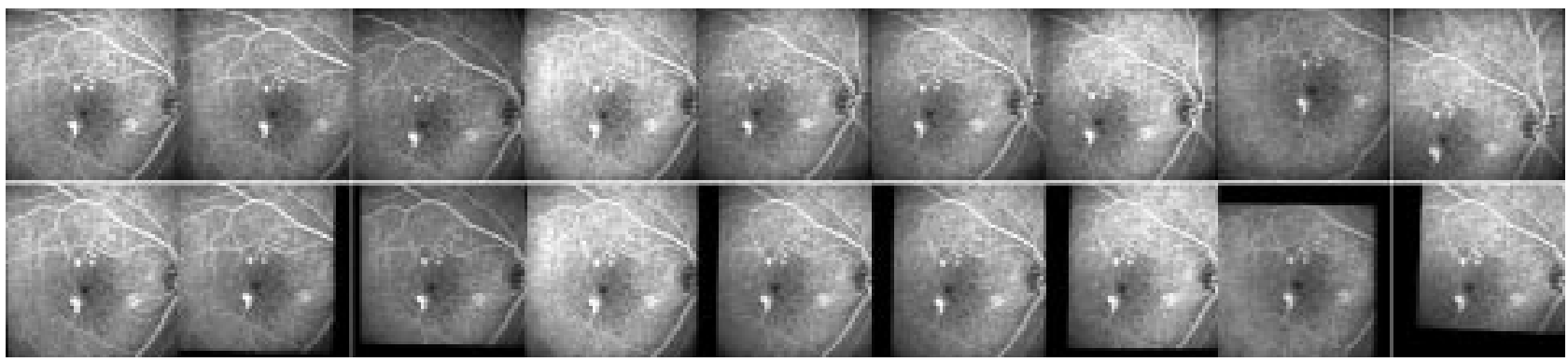

Fig. 14. (top) Original fluorescein sequence. (bottom) Registered sequence using the anchors methods. All images of the sequence are registered with the first image to remove eye motion. Notice how the patient moves his eye relative to the camera.

It is out of focus of this paper to review registration methods (see complete survey in [23]). We limit ourselves to the simple method we use, registration with anchors.

Let $\mathrm{I}_{b}, \mathrm{I}_{r}$ be two images. We wish to register $\mathrm{I}_{r}$ with $\mathrm{I}_{b}$, assuming $\mathrm{I}_{r}$ is obtained as result of a linear transformation from the basic image $\mathrm{I}_{b}$. There is then a vector $T$ and a matrix $R$ such that if $M_{b}$ and $M_{r}$ are matched points, respectively, in $I_{b}$ and $I_{r}$ then $M_{b}=T+R . M_{r}$. This equation being satisfied by $N$ pairs of control points, $N \geq 3$, the best affine transform is found by least squares based on correspondence on the set of matched points (see [1]). We illustrate in Fig. 14 the result of using this registration method on a sequence of retinal angiography during fluorescein examination. The control points were selected on the vascular tree. ${ }^{1}$

\section{B. Sequence of Fluorescein Images}

We consider in this section a sequence of images representing the progression of fluorescein in the vascular tree of a patient presenting drusen without confluences (see Fig. 14). At the various stages of the examination, we are interested in the evolution of the gray levels inside a particular drusen. Such tracking enables quantification of the diagnosis over time. Indeed, comparison of the results obtained during repetitive examinations makes it possible to consider the effect of a treatment for the patient. The images of our sequences are $256 \times 256$ with 256 gray levels. The examination lasts 20 min during which the doctor takes images at various intervals of time. Then, we carry out for each image of the sequence $I(t)$ the following processing.

- Smoothing of $I(t)$ by an average filter.

- Gray level normalization of $I(t)$ with same grey level range as reference $I(0)$ by a linear transformation.

- Registration of $I(t)$ with $I(0)$.

- Calculation of the adaptive contrast function $h(., t)$.

- Drusen segmentation as $h(., t)$ maxima of order $\beta$.

- Selection of drusen according to constraints (we use a structure of "linked list of linked list," see [1]).

- Tracking of different chosen drusen of the sequence.

- Output is the intensity evolution of the tracked drusen.

We show, in Fig. 15, the result of this approach on four images of the sequence. The images on left column show contours of the objects obtained after a segmentation using our algorithm of $h(x)$-maxima of order $\beta$. For all the images of the sequence we took as parameters: $\lambda=7, \gamma=7, \beta=5$. Notice that in this type of images, vessels are also bright areas, and they are

${ }^{1}$ We took three couples of points of junction on the vascular tree.

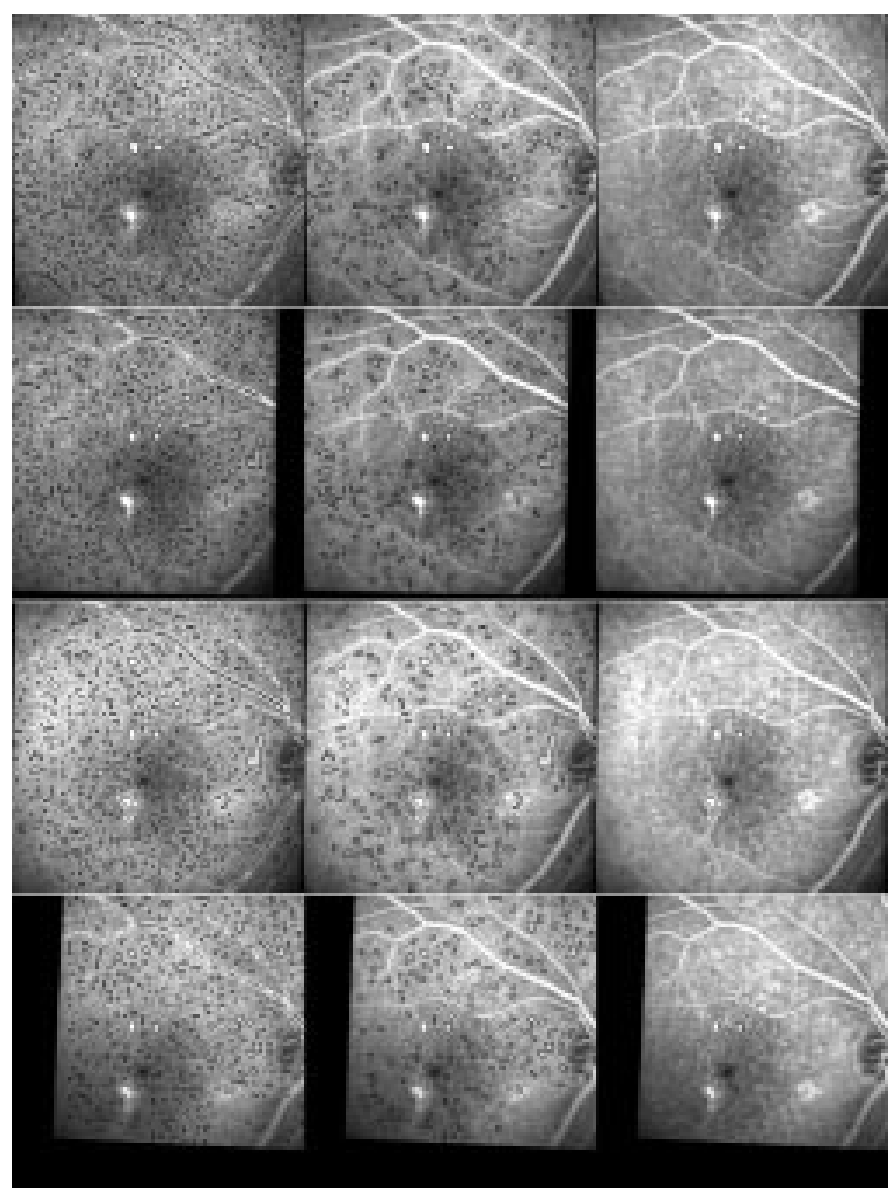

Fig. 15. (left) Our segmentation method on four registered images of the sequence of Fig. 14. (middle) After constraints of shape, contrast, and area. (right) Results of the tracking constraints.

detected using our segmentation approach. However, they are easily removed, as shown in the middle column images, after applying the constraints of shape, contrast and area described in Section III-G-3. The images on the right column illustrate the tracking of some chosen drusen. We specify that tracking is defined as the extra constraint that the tested object should be included in one of the initial rectangles defined around each tracked drusen chosen by the doctor.

We were able to track each drusen of the right column images. We show in Fig. 16 the evolution of the average intensity of the central drusen in our sequence of fluorescein examination. A similar evolution to that of Fig. 16 was established, but in a manual way, in the case of drusen sequence in [27]. 


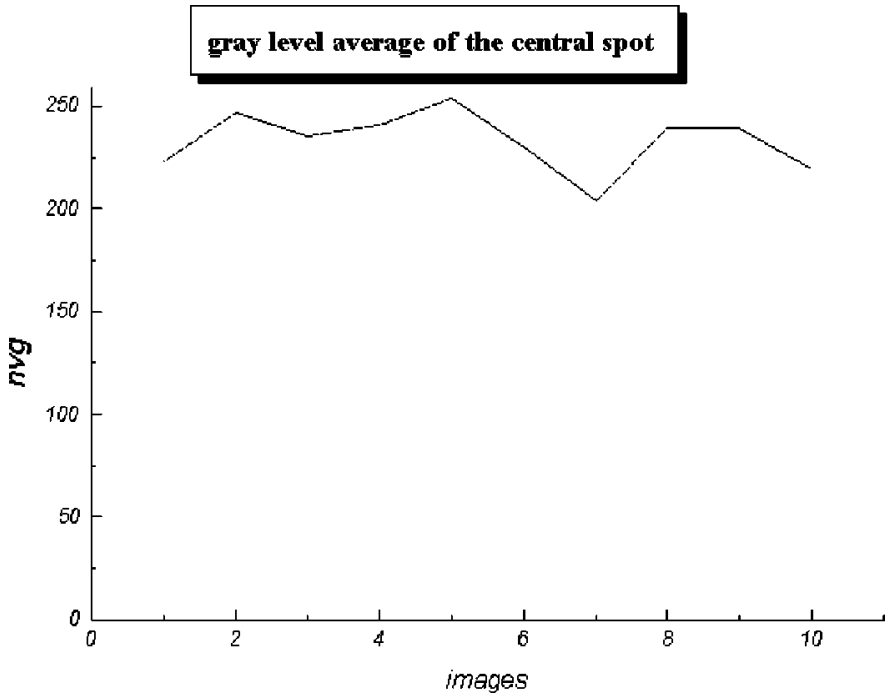

Fig. 16. Average intensity of a drusen in sequence of images.

\section{Classification and Rate of Aggravation of Drusen in the Course of Time}

We now use our segmentation tool as a preliminary stage for the tracking of drusen over time. The subjective diagnosis of the doctors evaluating the state of drusen for a patient could, thus, be replaced. Let $I_{t_{1}}$ and $I_{t_{2}}$ be two images such that: $t_{1} \leq t_{2}{ }^{2}$. We subject these two images to the same series of transformations described previously for registration and segmentation. We are interested to evaluate the difference in area occupied by drusen in the two images $I_{t_{1}}$ and $I_{t_{2}}$. We can calculate for each drusen of $I_{t_{2}}$ its match and its area change $d_{i}$ between the two times $t_{1}$ and $t_{2}$. We can also use our results to classify the types of evolution of drusen. For the classification of drusen between $t_{1}$ and $t_{2}$ we distinguished six classes as illustrated in Fig. 17.

- Class 1: increase or reduction in the area of drusen between times $t_{1}$ and $t_{2}$ without any modification in number.

- Class 2: the drusen vanishes at time $t_{2}$.

- Class 3: state of confluence between at least two drusen at time $t_{1}$ to give one drusen at time $t_{2}$.

- Class 4: division of a drusen at $t_{1}$ to at least two drusen at $t_{2}$.

- Class 5: creation of new drusen at $t_{2}$.

- Class 6: drusen not belonging to previous classes.

We used the relations of intersection between the objects of our segmentations at times $t_{1}$ and $t_{2}$, in order to classify the various types of drusen. For example, drusen $D_{1}$ of $I_{t_{1}}$ belongs to Class 1 , if and only if there exists a unique drusen $D_{2}$ of $I_{t_{2}}$ such that: $D_{1} \cap D_{2} \neq \emptyset$ and $\forall D_{j} \in I_{t_{1}}$ with $j \neq 1, D_{j} \cap D_{2}=\emptyset$. See [2] for more details. Our method is of course limited by the hypothesis that we have a correct segmentation for both images. In order to get similar results in the two images, they are normalized in gray level, and the same segmentation parameters are used. It may happen, however, that a drusen detected in the first image was removed in the second due to size or shape constraints. A way to improve this point would be to make a cooperative segmentation of the two images. Also, the constraints

${ }^{2} t_{1}$ and $t_{2}$ will be two dates of different examinations for example.
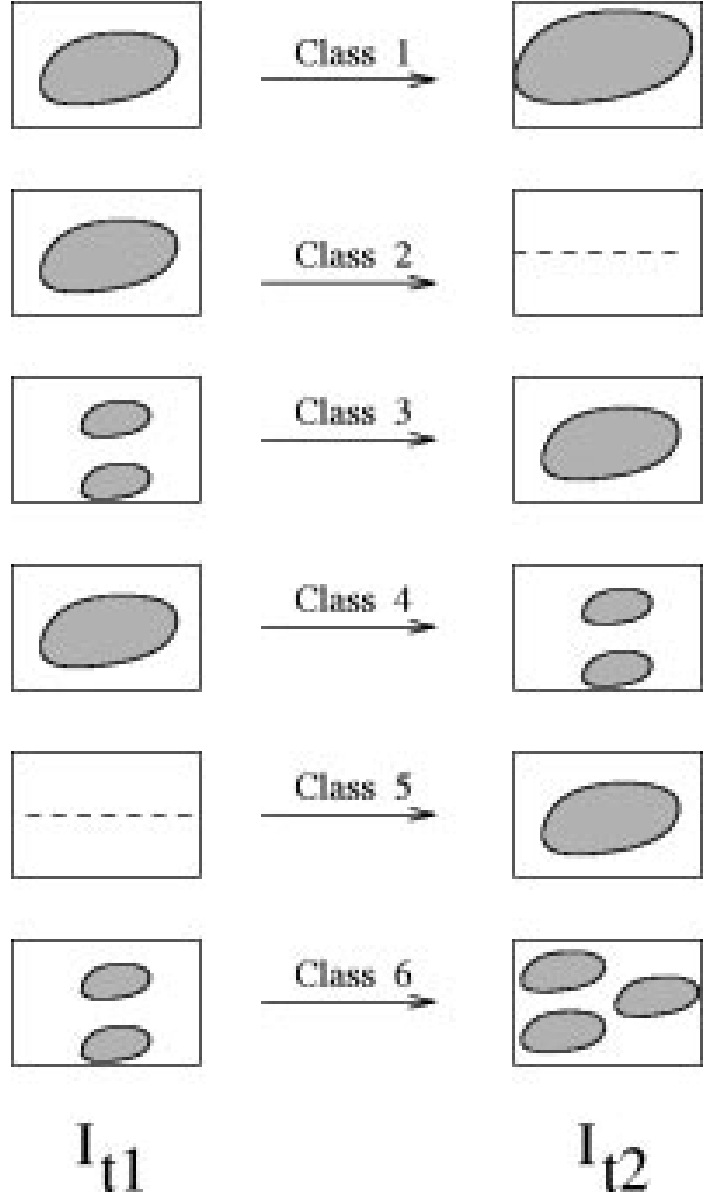

Fig. 17. Different classes of drusen.

may be applied only in conjunction with the presence of similar drusen in the other image. We show in Fig. 18 the images of an eye fundus presenting drusen for two different examinations. We applied our algorithm, as described above, to the central areas of the two images. We illustrate in Figs. 18 and 19 the results of our method of geometrical classification.

Our contribution, which consists of a geometrical classification of drusen allowed us to give to the doctor a perfectly automatic tool of recognition and appreciation of the various classes of drusen. Thus, we have the possibility of knowing with precision the area of each drusen of a given class, its space position, its average intensity and its match or matches, if they exist (case of the classes 1, 3, 4, and 6). These parameters make easier any later statistical study.

\section{CONCLUSION}

Understanding the behavior of drusen, and their quantification, over time is of primary importance for ophthalmologists. In this paper, we introduced new concepts based on mathematical morphology which are the maxima and minima of order $\lambda$. This is a new segmentation tool which enables very good segmentation of our images. This tool is based on the construction of a synthetic adaptive contrast function $h(x)$ from regional maxima and minima and by considering the connected components of the set $\left(f-\delta_{f, \beta}^{+\infty}(f-h)\right)_{1}$. Experimentation of this method on a set of images of different kinds and shapes of drusen is very 

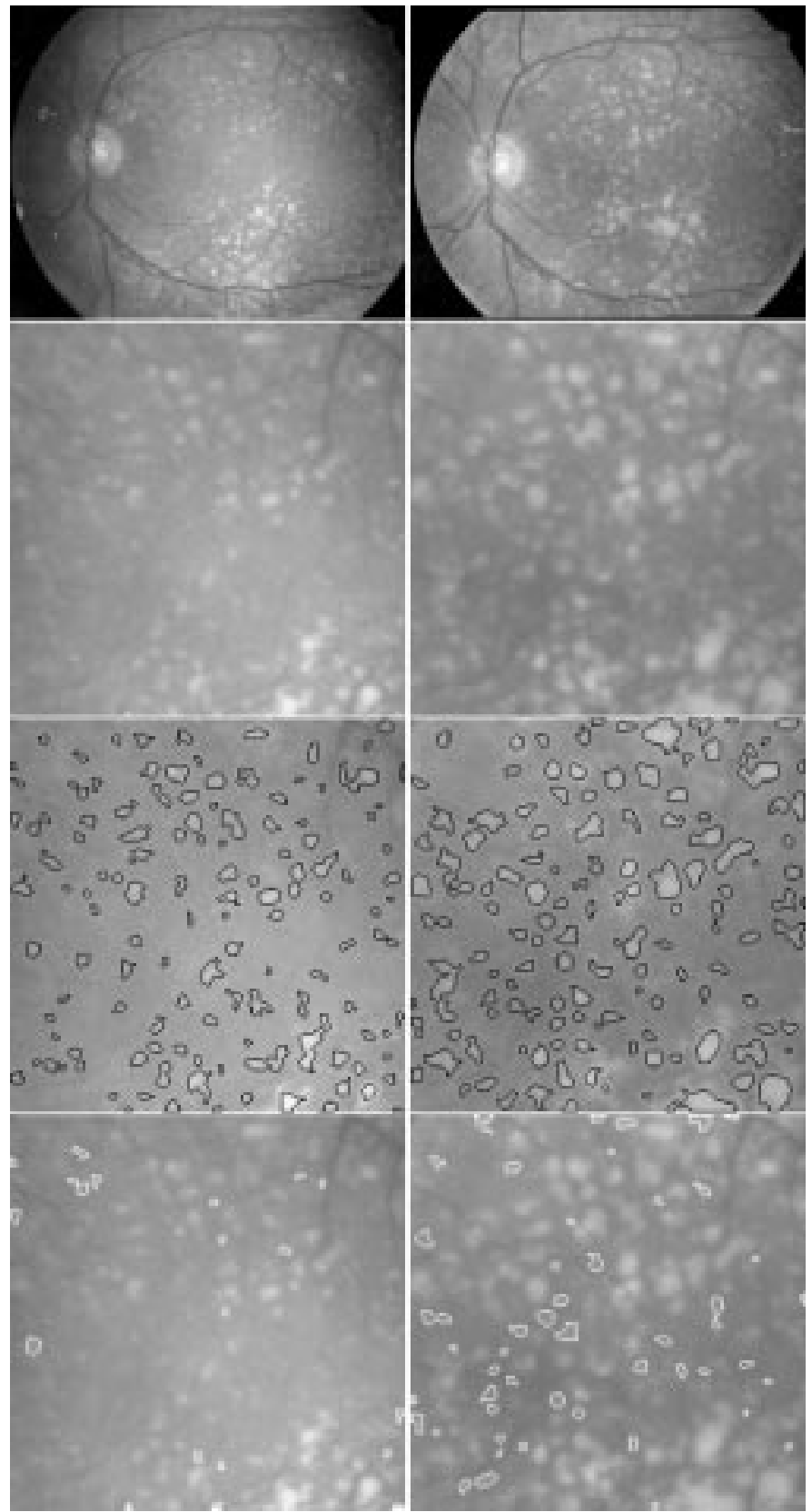

Fig. 18. (left) Drusen of a patient in 1983. (right) Same patient in 1988. From top to bottom, we show for both examinations the original image, a zoom on the central area, segmentation of the corresponding central area, examples of automatic classification (Class 2 (vanish) on the left, Class 5 (creation) on the right.

satisfying for the ophthalmologist. We extended this tool for the tracking and classification of drusen taken during a sequence of images. We illustrated by examples how our method of segmentation can be used in practice and contributes to the medical diagnosis of retinal angiography.

\section{APPENDIX \\ MATHEMATICAL MORPHOLOGY}

\section{A. Planar Structuring Element, Dilation and Erosion}

Let $K$ be a compact of $\mathbb{R}^{2}$. The planar structuring element associated to $K$ is the function $g_{K}$ defined by $g_{K}(x)=0$ if

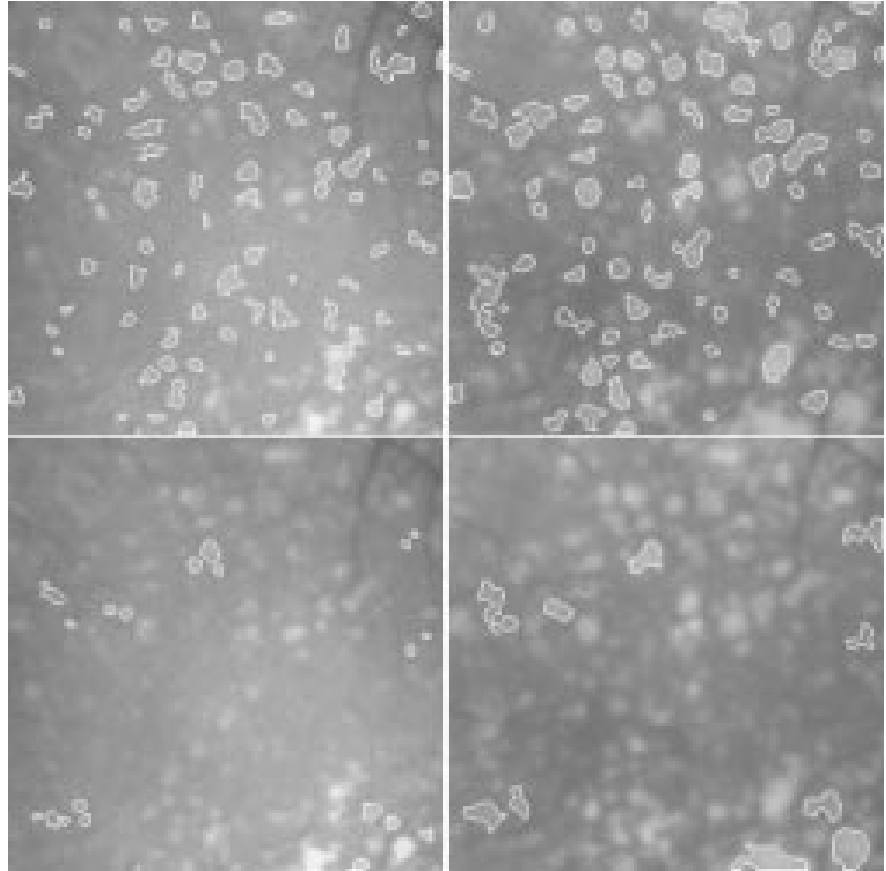

Fig. 19. Automatic classification of drusen on (left) $I_{t_{1}}$ and (right) $I_{t_{2}}$ on top for Class 1 (one-to-one correspondence) and on bottom for Class 3 (confluence).
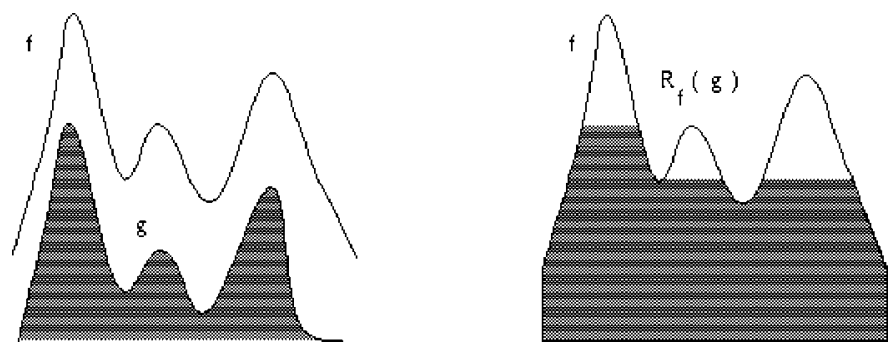

Fig. 20. The reconstruction function $R_{f}(g)$.

$x \in K$ and $-\infty$ else. Let $g_{K}$ be a planar structuring element, the dilation and erosion with $g_{K}$ are given, respectively, by: $f \oplus$ $g_{K}(x)=\sup _{y}\left\{f(y)+g_{K}(y-x)\right\}=\sup _{(y-x) \in K}\{f(y)\}$ and $f \ominus g_{K}(x)=\inf _{(y-x) \in K}\{f(y)\}$.

\section{B. Geodesic Dilation and Erosion}

Let $H$ be the unit ball. The geodesic dilation of a numerical function $f$ with respect to (or under) a function $g(f \leq g)$ is $\delta_{g}^{1}(f)=\inf [(f \oplus H), g]$. In the same way, the geodesic erosion of a numerical function $f$ with respect to (or above) function $g,(g \leq f)$ is $\varepsilon_{g}^{1}(f)=\sup [(f \ominus H), g]$. The composition formula of dilation and erosion in the case of the numerical functions give $\varepsilon_{g}^{r_{1}+r_{2}}(f)=\varepsilon_{g}^{r_{1}}\left(\varepsilon_{g}^{r_{2}}(f)\right)$ and $\delta_{g}^{r_{1}+r_{2}}(f)=$ $\delta_{g}^{r_{1}}\left(\delta_{g}^{r_{2}}(f)\right)$

It is very interesting to notice that in the case of planar structuring elements $K$, definitions above become: $\delta_{g}^{1}(f)(x)=\min \left\{\sup _{(y-x) \in K}[f(y)], g(x)\right\}$ and $\varepsilon_{g}^{1}(f)(x)=\max \left\{\inf _{(y-x) \in K}[f(y)], g(x)\right\}$

\section{Numerical Reconstruction}

We can define the reconstruction [29], [37] of a function $f$ starting from a function marker $g$, if $g \leq f$, as $R_{f}(g)=\delta_{f}^{+\infty}(g)$ 
where $\delta_{f}^{+\infty}$ is the limit when $r \rightarrow \infty$ of $\delta_{f}^{r}$ defined above. This operation allows the total or partial (according to $g$ ) construction of the image domes. In the case of valleys, we can consider the reconstruction by erosion, if $g \geq f$, we put then $R_{f}^{*}(g)=\varepsilon_{f}^{+\infty}(g)$.

\section{ACKNOWLEDGMENT}

The authors would like to thank the anonymous reviewers for their valuable suggestions. They would also like to thank Dr. $\mathrm{S}$. Prensky for his assistance with the final corrections of the manuscript. Drusen images are courtesy of Eye University of Creteil.

\section{REFERENCES}

[1] Z. Bensbeh, "New segmentation method in mathematical morphology based on geodesic reconstitution: Application to drusen extraction in ophthalmology digitized angiography imaging," Ph.D. dissertation, Univ. Paris-Dauphine, Ceremade, 1998.

[2] Z. Bensbeh, L. D. Cohen, G. Mimoun, and G. Coscas, "A New Approach for Geodesic Reconstruction in Mathematical Morphology and Application to Image Segmentation and Tracking in Ophtalmology," University Paris Dauphine, Ceremade, Tech. Rep. 0123, 2001.

[3] S. Beucher and F. Meyer, "The morphological approach to segmentation: The watershed transformation," Math. Morphol. Image Processing, pp. 433-482, 1993

[4] J. Canny, "A computational approach to edge detection," IEEE Trans. Pattern Anal. Machine Intell., vol. PAMI-8, pp. 679-698, Nov. 1986.

[5] V. Caselles, F. Catté, T. Coll, and F. Dibos, "A geometric model for active contours," Numerische Mathematik, vol. 66, pp. 1-31, 1993.

[6] V. Caselles, R. Kimmel, and G. Sapiro, "Geodesic active contours," Int. J. Comput. Vis., vol. 22, no. 1, pp. 61-79, 1997.

[7] L. D. Cohen, "Multiple contour finding and perceptual grouping using minimal paths," J. Math. Imag. Vis., vol. 14, no. 3, pp. 225-236, 2001.

[8] - "On active contour models and balloons," Comput. Vis., Graph., Image Processing: Image Understanding, vol. 53, no. 2, pp. 211-218, March 1991.

[9] L. D. Cohen and I. Cohen, "A finite element method for active contour models and balloons for 2-D and 3-D images," IEEE Trans. Pattern Anal. Machine Intell., vol. 15, no. 11, pp. 1131-1147, Nov. 1993.

[10] L. M. J. Florack et al., "Cartesian differential invariants in scale-space," J. Math. Imag. Vis., vol. 3, pp. 327-348, 1993.

[11] G. Gerig, G. Szekely, G. Israel, and M. Berger, "Detection and characterization of unsharp blobs by curve evolution," in Proceedings of Information Processing in Medical Imaging, ser. Computational Imaging and Vision. Norwell, MA: Kluwer, June 1995, pp. 165-176.

[12] W. R. Green, P. J. Mc Donnell, and J. H. Yeo, "Pathologic features of senile macular degeneration," Ophthalmology, vol. 92, pp. 615-627, 1985

[13] M. Grimaud, "La Géodésie Numérique en Morphologie Mathématique. Application à la Détection Automatique de Microcalcifications en Mammographie Numérique," Ph.D. dissertation, Ecole Nationale Supérieure des Mines de Paris, 1991.

[14] — "New measure of contrast: Dynamics," presented at the SPIE Image Algebra and Morphological Processing III, San Diego, CA, 1992.

[15] R. Jain, R. Kasturi, and B. G. Schunck, Machine Vision. New York: McGraw-Hill, 1995.

[16] M. Kass, A. Witkin, and D. Terzopoulos, "Snakes: Active contour models," Int. J. Comput. Vis., pp. 321-331, 1988
[17] K. R. Kenyon, A. E. Maumenee, and S. J. Ryan, "Diffuse drusen and associated complications," Amer. J. Ophthalmol., vol. 100, pp. 119-128, 1985

[18] T. Koné, "Recalage Automatique d'Images Angiographiques Retiniennes par Analyze Numérique d'Images: Application au Suivi de Sequences d'Images," Ph.D. dissertation, Univ. Paris XII Val-de-Marne, 1993.

[19] T. Koné, G. Mimoun, P. Bunel, G. Quentel, G. Soubrane, and G. Coscas, "A new algorithm for automatic detection of subretinal newvessels using digital angiography," Investigat. Ophthalmol. Visual Sci., vol. 33, no. 4, 1992.

[20] F. Leymarie and M. D. Levine, "Tracking deformable objects in the plane using an active contour model," IEEE Trans. Pattern Anal. Machine Intell., vol. 15, pp. 617-634, June 1993.

[21] T. Lindeberg, "Detecting salient blob-like image structures and their scales with a scale-space primal sketch: A method for focus-of-attention," CVAP, vol. 11, no. 3, pp. 283-318, 1993

[22] - Scale-Space Theory in Computer Vision. Norwell, MA: Kluwer, 1994.

[23] J. Maintz and M. A. Viergever, "A survey of medical image registration," Med. Image Anal., vol. 2, no. 1, pp. 1-36, 1998.

[24] F. Maisonneuve, "Extrema Régionnaux: Algorithme Parallèle,", Ecole des Mines de Paris, Tech. Rep. N781, 1982.

[25] R. Malladi, J. A. Sethian, and B. C. Vemuri, "Shape modeling with front propagation: A level set approach," IEEE Trans. Pattern Anal. Machine Intell., vol. 17, pp. 158-175, Feb. 1995.

[26] T. McInerney and D. Terzopoulos, "Deformable models in medical image analysis: A survey," MEDIA, Med. Image Anal., vol. 1, no. 2, pp. 91-108, November 1996.

[27] G. Mimoun, "Intéret de l'image numérisée dans le diagnostic et la classification des drusen maculaires,"Soc. Fr. Ophtalmologie, vol. 4, pp. $48-50,1989$.

[28] G. Mimoun, F. Coscas, T. Koné, P. Bunel, G. Soubrane, and G. Coscas, "Completely automatic overlay of fluorescein digital images," Investigat. Ophthalmol. Visual Sci., vol. 33, no. 4, 1992.

[29] L. Najman and M. Schmitt, "Geodesic saliency of watershed contours and hierarchical segmentation," IEEE Trans. Pattern Anal. Machine Intell., vol. 18, pp. 1163-1173, Dec. 1996.

[30] S. H. Sarks, D. V. Driel, L. Maxwell, and K. Killinsworth, "Softening of drusen and subretinal neovascularization," Trans. Amer. Ophthalmol. Soc. U.K., vol. 100 , pp. 414-422, 1980

[31] M. Schmitt and J. Mattioli, Morphologie Mathematique. Masson, France: Logique Mathematiques Informatique, 1994.

[32] J. Serra, Image Analysis and Mathematical Morphology. New York: Academic, 1988, vol. 2, Theoretical Advances.

[33] P. Soille, "Morphologie Mathématique : du Relief à la Dimensionalité-Algorithmes et Méthodes," Ph.D. dissertation, UCL, Louvain, Belgium, 1992.

[34] H. Tek and B. Kimia, "Image segmentation by reaction-diffusion bubbles," in Proc. 5th IEEE Conf. Computer Vision, Cambridge, MA, June 1995, pp. 156-162.

[35] C. Vachier, "Extraction de Caracteristiques, Segmentation, d'Image en Morphologie Mathématique," Ph.D. dissertation, Ecole Nationale Supérieure des Mines de Paris, Paris, France, 1995.

[36] L. Vincent, "Morphological area openings and closings for grey-scale images," presented at the NATO Workshop, Driebergen, The Netherlands, Sept. 1992

[37] - "Morphological grayscale reconstruction in image analysis," IEEE Trans. Image Processing, vol. 2, pp. 176-201, Feb. 1993

[38] L. Vincent and P. Soille, "Watersheds in digital spaces: An efficient algorithm based on immersion simulations," IEEE Trans. Pattern Anal. Machine Intell., vol. 13, pp. 583-598, June 1991.

[39] R. W. Young, "Pathophysiology of age related macular degeneration," Surv. Ophthalmol., vol. 31, pp. 291-306, 1987. 